\title{
Pion Production in the Quark Compound Bag Model of NN Scattering
}

\author{
T.-S. H. Lee
}

Physics Div., Argonne National Laboratory, Argonne, IL 60439-4843

\section{ABSTRACT}

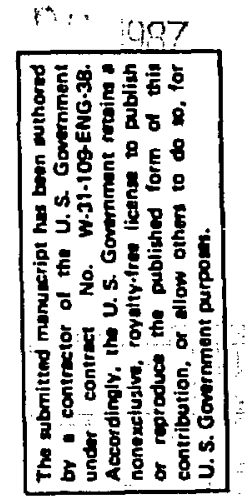

The quark compound Bag model of NN scattering is reviewed and extended to study pion-production from NN collisions.

The nucleon-nucleon interaction in the energy region below the pion production threshoid has been described by a phenomenological approach based on two assumptions: (a) The interaction at distances roughly larger than $0.7 \mathrm{fm}$ is described by meson-exchange mechanisms deduced either from the low order Feynman diagrams or the $\pi \mathrm{N}$ and $\pi \pi$ scattering data through the dispersion relations. (b) The short range part of the interaction is parameterized in terms of either mesonbaryon-baryon form factors or a computationally convenient local form. The success of this approach in describing low energy nuclear properties has motivated many theoretical efforts in extending the meson-exchange model to describe pion production processes. The outcome is the development of several $\pi N N$ models reviewed by $M$. Locher in this conference.

At this time, it is fair to say that none of the existing $\pi \mathrm{NN}$ models has achieved a complete success in descriking all relevant NN and $\pi d$ data. At least from the unitary meson-exchange $\pi N N$ calculations 1 by Dr. A. Matsuyama and myself, there is a strong indication that the use of the conventional parameterization of the short range interaction is the source of the problem. This is not very surprising since the hadrons are known to be composite particles made up of quarks, and their interactions at short distances must be energydependent and nonlocal. The conventional parameterization is clearly a very simplified description of the underlying multi-quark dynamics. It is a good approximation in the low energy region, but it should start to show its deficiencies as collision energy increases and must completely break down when the incoming wave length becomes much smaller than the size of the nucleon. To illustrate the limitation of the conventional meson-exchange model, I show in Fig. 1 a result from. our recent study 1 of four $\pi$ NN models. These models are constructed by using the subtraction method developed in Ref. 2 to extend four low energy NN potentials to include the $\Delta$-excitation and the coupling to $\pi N N$ and $\pi d$ channels. Qualitatively the differences between the considered models are mainly in their short range parameterizations. The Argonne V14 has a local Wood-Saxon form, and the Bonn 


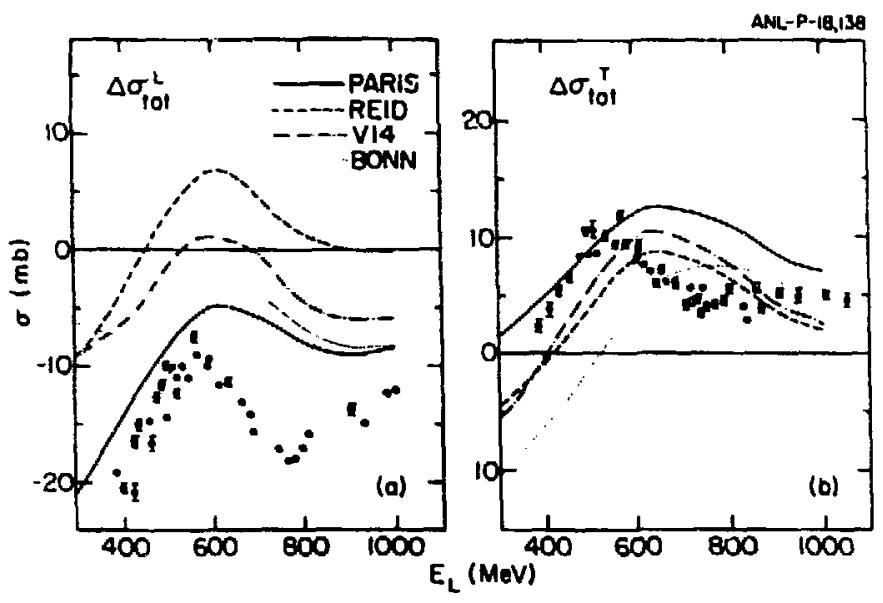

Figure 1

Total NN polarization cross sections calculated from four $\pi N N$ models are compared.

potential is highly nonlocal due to its momentum-space parameterization in terms of meson-baryon-baryon form factors. The Paris and Reid potentials are somewhat in between. Each model has only two free parameters: cutoff $\Lambda_{\pi}$ for defining the strength of the pion-exchange in the $N N++N \Delta$ potential and the $\pi N N++N N$ nonresonant pion production operator, and $E$ for extracting from the starting low energy $N N$ potential a $N N+N N$ interaction in the presence of the and $\Delta$ degrees of freedom. For each model, we adjust these two parameters to fit both the low energy ( $<300 \mathrm{Mev}$ ) NN phase shifts and the total NN reaction cross section up to $1 \mathrm{Gev}$. This procedure ensures that the resulting model can be as accurate as the conventional nuclear theory in describing low energy nuclear phenomena, while the strength of the corpling to $\pi N N$ channel is normalized to the gross feature of the pion production data. The accuracy of each model is then tested by examining their ability in describing the total polarization cross sections $\Delta \sigma_{T}^{\text {tot }}$ and $\Delta \sigma_{L}^{\text {tot }}$. It is clear from Fig. 1 that none of the constructed models can reproduce the strong energy-dependence of the data. In a recent paper, Popping, Sauer and $\mathrm{Zhang}^{3}$ also obtain results similar to our results based on the Paris potential. The subtraction method is also used in their construction of the model. This is why two calculations yield similar results.

The results shown in Fig. 1 suggest that it is necessary to develop a microscopic theory, or at least a new parameterization, of the short range interaction by using the information extracted from various QCD- 
motivated models of multi-quark systems. In this talk I will report the progress we have made by considering the information from the Bag model. First I will illustrate the general idea by showing how a model, called the Quark compound Bag model, can be quantitatively constructed to describe $\mathrm{NN} s$ wave scattering up to $1 \mathrm{Gev}$. Then I will describe the extension of the model to study pion production from NN collision.

Table I Six-quark Bag states

\begin{tabular}{ccccc}
\hline $\mathrm{J}^{+}$ & $\mathrm{I}$ & $\mathrm{MQ}(\mathrm{MeV})$ & $\mathrm{NN}$ Channel & $\mathrm{E}_{\mathrm{L}}(\mathrm{MeV})$ \\
\hline $0^{+}$ & 1 & $\sim 2200$ & ${ }^{1} \mathrm{~S}_{0}$ & $\sim 650$ \\
$1^{+}$ & 0 & $\sim 2100$ & ${ }^{3} \mathrm{~S}_{1}$ & $\sim 600$ \\
$2^{+}$ & 1 & $\sim 2300$ & ${ }^{1} \mathrm{D}_{2}$ & $\sim 900$ \\
\hline
\end{tabular}

The quark compound Bag model is motivated by the observation that the mass spectrum calculated from the Bag model for a six-quark system is reachable in the intermediate energy NN collision. In Table I, I list the lowest Bag states cisculated by Mulders ${ }^{3}$ and the needed incident nucleon laboratory energies for producing the same total energies in NN collisions. Table I suggests that if the Bag model is a good approximation to QCD, then these "bare" six quark states must play some roles in determining two-nucleon interaction at least in the regions near the indicated collision energies. The coupling of these sixquark systems to the $N N$ and $\pi N N$ channels will determine their physical mass and their lifetime. If the imaginary part of the resulting physical mass is small then the six-quark system will correspond to a resonance in NN collision. If its imaginary part is very large, then it merely is a microscopic description of short range interaction.

Following the approach first proposed by Simonov, ${ }^{5}$ the six-quark Bag dynamics is included in NN scattering by postulating that the total wave function of the system has two components

$$
\left|\phi_{B}\right\rangle=\left|\phi_{B}\right\rangle+C_{Q}(E)|Q\rangle
$$

where $\left|{ }_{E}\right\rangle$ describes a two-cluster $N N$ channel, and $|Q\rangle$ is the confined six-quark state. By using the standsrd coupled channel method, it is easy to show that the Schrodinger equation for the relative wave function $\chi_{\mathrm{E}}(x)$ in NN channel is of the following form 


$$
\left[-\frac{1}{2 \mu} \vec{\nabla}^{2}+V(\vec{r}, E)-E\right] \chi_{E}(\vec{r})=-\frac{f_{Q}^{*}(\vec{r}, E)}{E-M_{Q}} \int f\left(\vec{r}^{\prime}, E\right) \chi_{E}\left(\vec{r}^{\prime}\right) d \vec{r}^{\prime}
$$

where

$$
\begin{aligned}
& V(\vec{r}, E)=\left\langle\Phi_{E}|(B-E)| \Phi_{E}\right\rangle \\
& u_{Q}=\langle Q|H| Q\rangle \\
& f_{Q}(\vec{r}, E)=\left\langle\Phi_{E}|(H-E)| Q\right\rangle
\end{aligned}
$$

The dynamics of the model is contained in the matrix elements of Eq. (3). We use the well-studied meson-exchange model, such as the Paris potential, to define the interaction $\mathrm{V}(\vec{r}, \mathrm{E})$ at large distances. Following the notion of the asymptotic freedom we assume that no interaction at short distances should be present in either the two-cluster NN channel or a six-quark channel. The short range interaction is entirely due to the transition Eq- (3c) from the two-cluster NN channel to the sixquark confined state. In practice we assume that

$$
\begin{aligned}
V(\vec{r}, E) & =0 & & r \leq d \\
& =V_{\text {Paris }}(\vec{r}, E) & , & r \geq d
\end{aligned}
$$

The parameter d must be related to a QCD length scale which determines how the meson-exchange emerges from the quark-gluon dyamics. Obviously, this length parameter and Bag excitation matrix element $f_{Q}(\vec{r}, E)$ can only be determined phenomenologically.

It is convenient to parameterize the Bag excitation in a Taylor expansion around the Bag mass $\mathrm{M}_{\mathrm{Q}}$

$$
f_{Q}(\vec{r}, E)=C f_{0}\left(r, u_{D}\right)+\sum_{n \geq 1} X_{n}\left(E-M_{Q}\right)^{n_{f}} f_{n}\left(r, M_{D}\right)
$$

It was first pointed out by Simonov ${ }^{5}$ that if the energy independent term $f_{0}$ is localized at a distance $b$, then the solution of the Schrodinger Eq. (2) can reproduce the P-matrix interpretation of the Bag model. More importantly, the resulting wave function of the twonucleon component $\chi_{\mathrm{E}}(\mathrm{r})$ becomes completely excluded from the Bag region when the total collision energy is exactly equal to the Bag mass. This is illustrated in Fig. 2 taken from the work by Fasano 


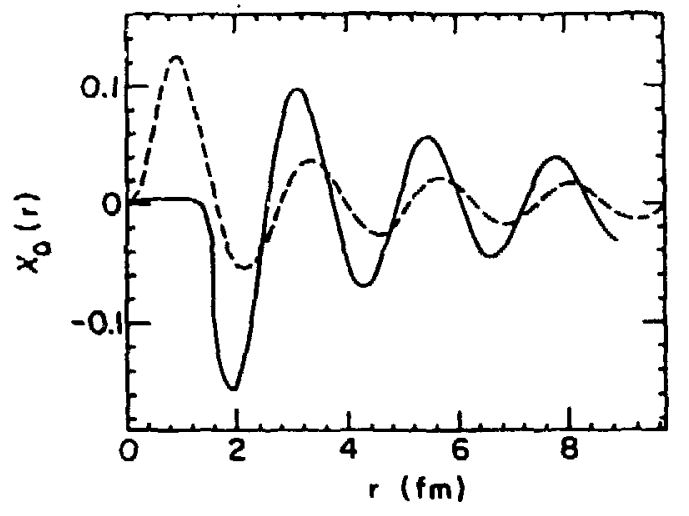

Figure 2

The ${ }^{1} \mathrm{~S}_{0}$ scattering wave functions at the Bag energy $E=M_{Q}=2159 \mathrm{MeV}$ calculated from the Paris potential (-) and the quark compound Bag model (-) are compared.

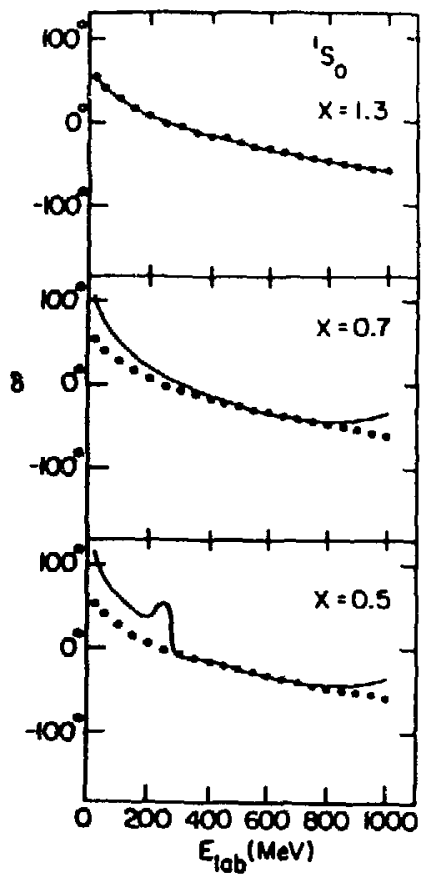

Figure 3

Effect of the energy-dependent term of Eq. (5) on the fit to ${ }^{1} S_{0}$ phase shifts.

and myself. ${ }^{6}$ This means that at the Bag energy the system has a two-phase picture in which the short range part is completely determined by the quark configuration. For this reason the parameter b wast be about the size of the considered six-quark Bag. Following the P-matrix interpretation by Jaffe and Low $_{8}{ }^{7}$ we use $b=1.4 \mathrm{fm}$ as extracted in the analysis by Bakker et al. Note that at other energies both the NN component and six-quark component can exist at short distances. Our model is therefore conceptually not very different from the ccexistence model developed by Yamauchi and Wakamatsu.

In fitting the NN phase shift up to $1 \mathrm{Gev}$, we need to include the energy dependent terms of the Bag excitation form factor defined by Eq. (5). In the study of NN 8 wave scattering, it was found by the ITEP $g r \cdots p^{10}$ and yerified later by Fasano and myself that it is essentia: keep the leading energy-dependent term. In Fig. 3, I show our fit to the ${ }^{1} S_{0}$ phase shift and the importance of the energydependent term. By reducing its strength from the fitted value $x=1.3$ 
to 0.5 the model produces a resonance which is of course in contradiction with the data. Asother important finding from our study is that no fit can be obtained if the background meson-exchange interaction $V(\vec{r}, E)[E q$. (4)] is cut off at a distance larger than $d=0.7$ fm. This is somewhat undesirable since the physical meaning of the meson-exchange interaction inside the Bag region is not clear. Perhaps it can be justified from the point of view of the collision between two cloudy Bags. In any case, it is more appropriate to consider it a phenomenological procedure and needs to be clarified in the future.

The s-wave study is only for the purpose of understanding the dynamical content of the Bag excitation mechanism. To resolve the $\pi N N$ problem such as that illustrated in Fig. 1, we need to incorporate the Bag excitation mechanism into the unitary $\pi N N$ formulation. This was accomplished by Matsuyama and myself in Ref. 11 . We now apply our formulation to include the Bag excitation in the study of pp + png ${ }^{+}$reaction.

Our approach can be illustrated by considering the coupling of the $2^{+}$Bag state shown in Table I to the most important ${ }^{5} \mathrm{~S}_{2} \mathrm{N \Delta}$ channel which is known to be the main source of pion production. In the meson-exchange $\pi N N$ model, the $N \Delta$ state is excited by the exchange of $\pi$ and $\rho$ mesons between two nucleons. In constructing the quark compound Bag model we extend Eq. (4) to also set the transition $\mathrm{NN}+\mathrm{N} \Delta$ interaction to zero inside the region $\mathrm{r}<\mathrm{d}$. The Bag excitation is correspondingly described by extending the form factor Eq.5 to include the decay into a NA channe!. For simplicity we assume that all energy dependent terms of Eq. (5) have the same $r-$ dependence. For a given choice of the rainge parameters, $d$ for the cutoff of $V(\vec{r}, E)$ and the Bag size $b$, the coefficients $c$ and $x_{n}$ of the Bag excitation form factor [Eq.( 5)] are determined in a $\chi^{2}$-fit to the NN ${ }^{1} D_{2}$ phase shift. The needed numerical procedures have been welldeveloped in Refs. 1 and 6 . It is found that the fit can be obtained only when at least the $\left(\mathrm{M}_{\mathrm{Q}}-\mathrm{E}\right)^{2}$ term is included. This is reasonable since in the pion production channel the energy dependence has to be much stronger than the predominantly elastic s-wave NN channel.

From our s-wave study (Fig. 2), we expect that the short range part of the $N N$ and ND components calculated from our quark compound Bag model are very different from that of the usual mesonexchange model. We therefore expect that the differences between two models in describing pion production processes can be best studied in the kinematic region where the relative momentum of the final $N \Delta$ state can be zero, and hence the transition from a initial NN state to a final $N \Delta$ state can involve the largest momentum transfer the reaction can provide. To see the $\Delta$ effect, the invariant mass of the outgoing $\pi \mathrm{N}$ pair must be $1236 \mathrm{Mev}$ at the point where the relative 


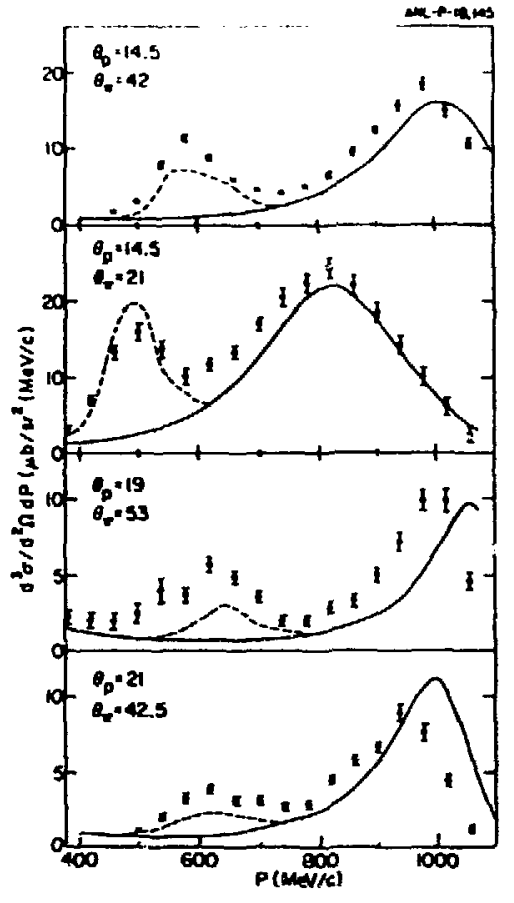

Figure 4

Results of $\mathrm{pp}+\mathrm{pp} \pi^{+}$from Ref. 12.

NA momentum is zero. This can be achieved by $650 \mathrm{Mev}$ incident nucleon laboratory energy. It is easy to show that by choosing $\theta_{p}=19.3^{\circ}$ for the outgoing proton and $\theta_{\pi}=69.3^{\circ}$ for the outgoing $\pi^{+}$ we can obtain the desirable kinematics in pp+pnat reaction. We will only present our result for this kinematics.

The cross section of
$p p \rightarrow p p \pi^{+}$is calculated by using the unitary approach developed by Dr. Matsuyama and myself. ${ }^{12}$ Within the meson-exchange model, we are

able to describe the main features of the data both in the shape and the absolute magnitude of the cross section. But it is also clear that the meson-exchange model has difficulties which cannot be easily resolved within the conventional approach stated in the beginning of this talk. In Fig. 4, I show some of our results. We now show that the quark compousd Bag model gives a very different description of pion production and hence offers a possible way to resolve the problem.

Following our unitary formulation, the amplitude of $\mathrm{NN}+\mathrm{NN \pi}$ can be written as

$$
\begin{aligned}
T_{\pi N N, N N}(E) & =h_{\pi N, \Delta} G_{N \Delta}(E) X_{N \Delta, N N}(E) \\
& +\nabla_{d} G_{\pi d}(E) X_{\pi d, N N}(E)
\end{aligned}
$$

where

$$
\begin{aligned}
& X_{N \Delta, N N}(E)=\left[1+X_{N \Delta, N N}(E) G_{N \Delta}(E)\right] T_{N \Delta, N N}(E) \\
& X_{\pi d, N N}(E)=X_{\pi d, N \Delta}(E) G_{N \Delta}(E) T_{N \Delta, N N}(E)
\end{aligned}
$$


The first term of Eq. (6) is the produrtion due to the direct decay of the $\Delta$, while the second term contains the final state interaction. It is clear that the most important dynamics is contained in the transition amplitude $\mathrm{T}_{\mathrm{NA}, \mathrm{NN}}$. Within the conventional meson-exchange model, it is determined from $N N+N \Delta$ transition potential and $N N$ scattering wave function

$$
T_{N \Delta, N N}(E)=V_{N \Delta, N N} n_{N N}^{\left(+l_{E}\right)}
$$

When the coupling to a six-quark system is included, we have

$$
\begin{aligned}
T_{N \Delta, N N}(E)+ & {\left[V_{N \Delta, N N}+f_{N \Delta, Q} \frac{1}{E-M_{Q}-\Sigma_{Q}} f_{Q, N N}\right] \Omega_{N N}^{(+)}(E) } \\
& \left.+f_{N \Delta, Q \frac{E}{E-M_{Q}-\Sigma_{Q}(E)}} f_{Q, N \Delta} G_{N \Delta}(E) T_{N \Delta, N N}(E)\right]
\end{aligned}
$$

where $\Sigma_{O}$ is the self-energy of the six-quark system due to its coupling to the NIN and $N \Delta$ channels.

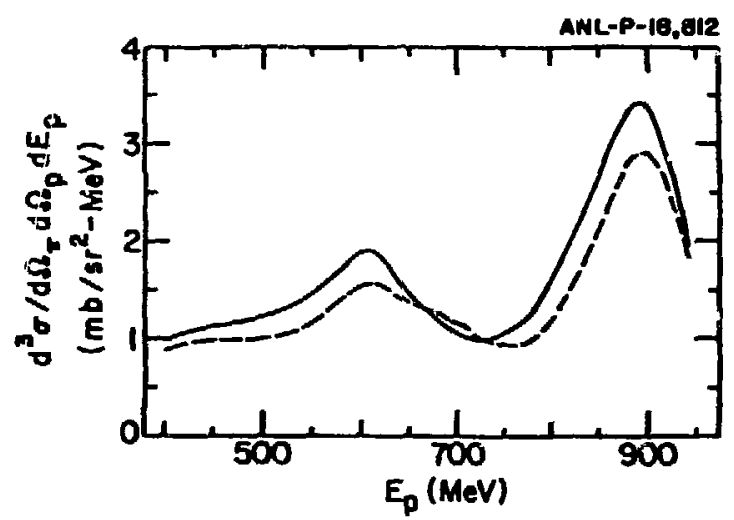

Figure 5 $p p+p p \pi^{+}$cross section at $\mathrm{E}=650 \mathrm{MeV} \theta_{\mathrm{p}}=19.3^{\circ}$, $\sigma_{\pi}=69.3^{\circ}$. The solid curve is from the conventional mesonexchange model of Ref. 12 . The dashed curve is from the quark compound Bag model.

Clearly two models have different production mechanisms, although both fit the same NN phase shift. In Fig. 5 we show their difference in predicting the cross section of pp+pn $\pi^{+}$at the kinematics, $\theta_{p}=19.3^{\circ}$, $\theta_{\pi}=69.3^{\circ}$ and $E=650 \mathrm{Mev}$, where the relative $N \Delta$ momentum can be zero and hence the $N \Delta$ s-wave plays the most important role. The results shown in Fig. 5 clearly indicates that the study of pion production can be useful in examining the difference between the conventional parameterization and the quark compound Bag model.

Within the quark compound Bag model, the parameterizations are still not very restricted. For example, the parameter $b$ at which the Bag excitation takes place is not well defined, due to the uncertainties 


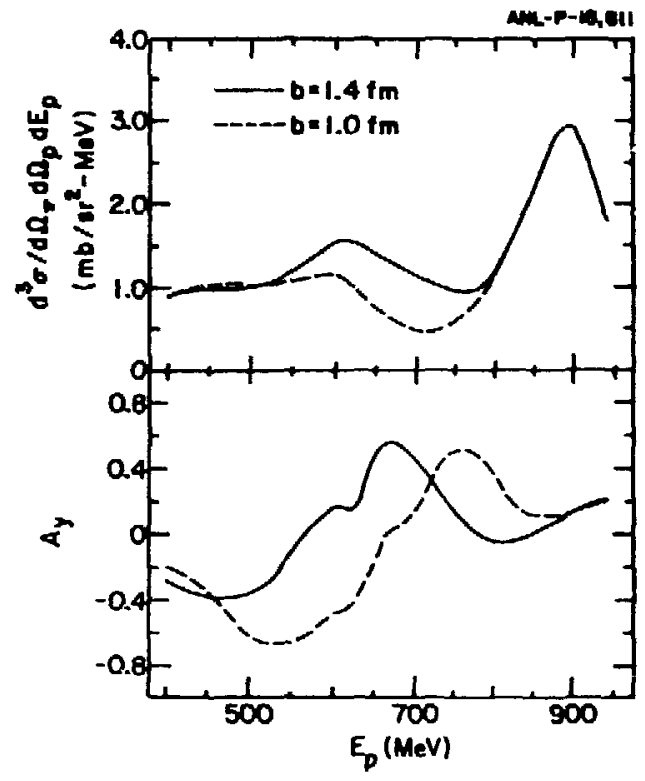

Figure 6 $p p+p p \pi^{+}$cross section at $\mathrm{E}=650 \mathrm{MeV} . \theta_{\mathrm{p}}=19.3^{\circ}$, $\theta_{\pi}=69.3^{\circ}$, calculated from quark compound Bag model with the Bag size $b=1.4 \mathrm{fm}$ and $1.0 \mathrm{fm}$.

in the Bag model calculations. The study of $\pi$ production can help to clarify these uncertainties. To illustrate this, we construct two models with $b=1.4$ and $1.0 \mathrm{fm}$. With appropriate choices of $c$ and $x$ 's, both models give the same fit to ${ }^{1} D_{2}$ phase-shifts. Their predictions on $\pi$ production are compared in Fig. 6. Clearly the differences are very dramatic.

In conclusion, we have developed a quark compound Bag model for describing $\pi N N$ system. It is shown that the mode! gives a very different description of $N N$ wave function in the region where the Bag state can be excited. The study of $\mathrm{NN}+\mathrm{NN} \pi$ process is very useful to test the model. In the future we need to consider all partial waves so that the validity of the model can be tested realistically. The experimental data at $650 \mathrm{Mev}$ is particularly useful for distinguishing the quark compound Bag model from the conventional meson-exchange model. We hope more measurements can be made in the near future.

This work supported by the U.S. Department of Energy, Nuclear Physics Division, under contract W-31-109-ENG-38.

\section{REFERENCES}

1. T.-S. H. Lee and A. Matsuyama, to be published in Phys. Rev. C.

2. T.-S. H. Lee, Phys. Kev. Lett. 50 (1983) 157; Phys. Rev. C 29 (1984) 195.

3. H. Popping, P. U. Sauer and S. Zhang, preprint (University of Hannover 1987). 
4. P. J. Mulders, A. T. Aerts and J. J. deSwart, Phys. Rev. D 21 (1980) 2653.

5. Yu. A. Simonoy, Phys. Lett. 107B (1981) 1.

6. C. Fasano and T.-S. H. Lee, to be published in Phys. Rev. C.

7. R. L. Jaffe and F. E. Low, Phys. Rev. D19 (1979) 2105.

8. B. L. G. Bakker, I. L. Grach and I. M. Narsdetshii, Nucl. Phys. A424 (1984) 563.

9. Y. Yamauchi and M. Wakamatsu, Nucl. Phys. A457 (1986) 621.

10. Yu. S. Kalashnikova, I. M. Narodetskii, Yu. A. Simonov and A. I. Veselov, Phys. Lett. 155B (1985) 217; I. L. Grach, Yu. S. Kalashnikova and I. M.narodetskii, preprint ITEP86-196 (1986).

11. T.-S. H. Lee and A. Matsuyama, Phys. Rev. C 32 (1985) 516.

12. A. Matsuyama and T.-S. H. Lee, Phys. Rev. C 34 (1986) 1900.

\section{DISCLAIMER}

This report was prepared as an account of work sponsored by an agency of the United States Government. Neither the United States Government nor any agency thereof, nor any of their employees, makes any warranty, express or implied, or assumes any legal liability or responsibility for the accuracy, completeness, or usefulness of any information, apparatus, product, or process disclosed, or represents that its use would not infringe privately owned rights. Reference herein to any specific commercial product, process, or service by trade name, trademark, manufacturer, or otherwise does not necessarily constitute or imply its endorsement, recommendation, or favoring by the United States Government or any agency thereof. The views and opinions of authors expressed herein do not necessarily state or reflect those of the United States Government or any agency thereof. 